\title{
Real-World Adverse Effects of Capecitabine Toxicity in an Elderly Population
}

\author{
Michiel W. H. van Beek ${ }^{1,2}$ - Monique Roukens ${ }^{3,7}$ - Wilco C. H. Jacobs ${ }^{4}$ Johanna N. H. Timmer-Bonte ${ }^{3}$. \\ Cees Kramers ${ }^{1,5,6}$
}

Published online: 22 June 2018

(c) The Author(s) 2018

\begin{abstract}
Background Few studies have assessed the safety and effectiveness of the numerous available chemotherapeutic therapies for geriatric oncology patients. Most safety studies are conducted in large trials, and there is some uncertainty surrounding whether the results would be the same in typical daily use.

Objective This retrospective study aims to assess the adverse effects of real-world capecitabine use in elderly patients.

Methods We reviewed the records of patients treated with capecitabine in an oncology department of a University Clinic in Nijmegen, The Netherlands. We scored adverse effects such as hand-foot syndrome and diarrhea, and dosage adjustments and the reasons for them. In total, 132 patients were included, 69 of whom were aged 70 years or below (mean age: 57 years), while 63 were aged older than 70 years (mean age: 74 years).

Results Patients aged over 70 years experienced more serious adverse effects than younger patients. Grade 2 or 3 hand-foot syndrome toxicity was experienced by $20.2 \%$ of patients aged younger than 70 years and by $34.9 \%$ of patients older than 70 years $(p=0.059)$. Grade 2,3 , or 4 diarrhea was experienced by $17.4 \%$ of the patients aged younger than 70 years but by $31.7 \%$ of the patients aged older than 70 years $(p=0.044)$. Dosage was adjusted for $27 / 69$ patients in the younger group and $52 / 63$ patients in the older group $(p=0.001)$.

Conclusion The difference in observed adverse effects cannot be the sole explanation for the high incidence of observed dose adjustments. A prospective follow-up study of elderly patients using capecitabine outside clinical trials is needed to evaluate the optimum balance between adverse effects and efficacy.
\end{abstract}

Michiel W. H. van Beek

Michiel.v.beek@ catharinaziekenhuis.nl

1 Department of Pharmacology-Toxicology, Radboud University Medical Center, Nijmegen, The Netherlands

2 Department of Clinical Geriatrics, Catharina Hospital, Michelangelolaan 2, 5623 EJ Eindhoven, The Netherlands

3 Department of Medical Oncology, Radboud University Medical Center, Nijmegen, The Netherlands

4 The Health Scientist, The Hague, The Netherlands

5 Department of Internal Medicine, Radboud University Medical Center, Nijmegen, The Netherlands

6 Department of Clinical Pharmacy, Canisius Wilhelmina Hospital, Nijmegen, The Netherlands

7 Department of Clinical Pharmacy, Radboud University Medical Center, Nijmegen, The Netherlands

\section{Key Points}

This study shows that when used in daily practice:

The incidence of capecitabine-related adverse effects in cancer patients aged $>70$ years of age differs from younger patients

Capecitabine results in more (severe) diarrhea in patients aged 70 years or older

Older patients receive more frequent dose adjustments than younger patients

Dose adjustments are seen in a higher proportion of older patients than can be accounted for by severe adverse effects 


\section{Introduction}

As people are living longer, the incidence of cancer is increasing. Today, more than half of patients with cancer are over 70 years of age [1], and are often given either curative or palliative cancer treatments. Most previous studies on systemic cancer treatments have excluded or underrepresented elderly patients; for example, about 50\% of patients with colon cancer are aged 70 years or above, but represented just $16 \%$ of the patients who participated in trials [2]. Because data concerning elderly patients with cancer are scarce, it is very difficult to advise on adjuvant chemotherapy for patients over 70 years of age; thus, this decision is frequently based on the intuitive judgment of the physician rather than on evidence-based guidelines.

Capecitabine is an oral chemotherapy agent approved in 2001, which has been proven effective for, and is frequently used in, curative and palliative colon cancer, gastric cancer, and breast cancer regimens $[3,4]$. Its use has increased because patients prefer this oral administration over the 5-day intravenous 5-fluorouracil/FA therapy standard of care (Mayo Clinic regimen), which has a comparable efficacy [5, 6]; however, the adverse effects of these two treatment types are different [7]. Capecitabine is an oral progenitor drug that is converted to 5-fluorouracil in the body, causing cell death by RNA- and DNA-related mechanisms [8]. In adults, capecitabine has a bioavailability of $\sim 100 \%$ with a maximum plasma concentration of $3.9 \mathrm{mg} / \mathrm{L}$, time to maximum plasma concentration of $1.5-2 \mathrm{~h}$, and area under the plasma concentration-time curve of $5.96 \mathrm{mg} \cdot \mathrm{h} / \mathrm{L}$. The predominant route of elimination is renal, and dosage reduction of $75 \%$ is recommended in patients with creatinine clearance of $30-50 \mathrm{~mL} /$ $\min$. The drug is contraindicated if creatinine clearance is $<30 \mathrm{~mL} / \mathrm{min}$ [9]. The incidence of adverse effects such as diarrhea, stomatitis, nausea, and neutropenia was lower in the patients treated with capecitabine than with 5-fluorouracil [4]; however, the rate of hand-foot syndrome (HFS) was higher [10]. The pathophysiology of HFS is complex and not fully understood [11].

In most studies, elderly patients were found to experience more adverse events related to the use of capecitabine [7, 8, 12], although one study [13] showed no difference in the incidence of grade 3 or 4 adverse events between patients aged older than 80 years and the overall population. The impact of these adverse effects on patient quality of life, treatment compliance, and disease-related outcomes in the elderly is largely unknown. In daily practice, disease-related outcomes might be influenced by dose adjustments due to toxicity, which are very common, although some studies indicated that the consequent reduction in efficacy was not very high [10]. The primary aim of this study was to compare the adverse effects of real-world capecitabine treatments in patients older and younger than 70 years of age. Further, we compared the incidence of chemotherapy dose adjustments and the reasons for them.

\section{Methods}

The Radboud University Medical Center Institutional Review Board concluded that the study did not fall within the scope of the Medical Scientific Research Act because it was a retrospective observational study, without intervention and with no burden for the participants. Patients who had been prescribed capecitabine between January 2007 and December 2013 were retrospectively identified from the records of the hospital's outpatient pharmacy, which is responsible for outpatient chemotherapy. All patients were linked to data stored in electronic patient files of the oncology department of the Radboud University Medical Center (electronic patient database). Data on the chemotherapy, dosing, and adverse effects were collected, as well as patient characteristics. Patients with incomplete data were excluded. In this study, only Xeloda ${ }^{\circledR}$ was used.

The primary outcomes were the occurrence and severity of capecitabine-related toxicity. Secondary outcomes were dose adjustments and their reasons. The toxicity and severity of HFS and diarrhea were classified according to the National Cancer Institute Common Terminology Criteria for Adverse Events Version 3.0. The frequency (number) of dose adjustments was assessed, as were the reasons for these adjustments and any early ending of therapy. Adverse effects in cycle 1 were assessed just prior to the start of cycle 2 and this process was continued. The assessment was performed by the treating physician, one of eight academic medical oncologists in accordance with standard practice. Patients with upfront dosage reductions of capecitabine were not included in the study.

Adverse effects were divided into two groups: no or mild adverse effects (grade 0 or 1), and (more) severe or lifethreatening adverse effects (grade 2-4). Age was categorized into two groups: 70 years or below, and over 70 years. This cut-off at the age of 70 years is frequently used in geriatric and geriatric oncology literature $[14,15]$. Missing data were not replaced or imputed. Differences in the incidence and severity of HFS, diarrhea, and neutropenia were analyzed using a Chi square analysis performed in SPSS Version 16.

\section{Results}

In total, 149 patients who received capecitabine from the hospital pharmacy in the inclusion period were identified. Their background characteristics were extracted from the 
electronic patient file. We excluded 17 patients with incomplete data on adverse effects, resulting in 132 (89\%) patients being included, 77 of whom were men and 55 were women. The age of the patients varied between 47 and 87 years at the start of the capecitabine treatment. The patients were divided into two study groups; patients older than 70 years (63 patients) and patients aged 70 years or below (69 patients). The patient characteristics are shown in Table 1. The two groups initially received similar starting doses of capecitabine, and had similar Karnofsky Performance Scale Index scores, intentions of treatment, tumor types, and additional chemotherapies. Differences were detected in the renal function, Charlson Comorbidity Index scores, polypharmacy, and sex distributions of the two groups.

The distribution of adverse effects across the two age groups is given in Table 2. There was no statistical difference in the frequency of HFS (all grades) in the older patients (27/63 patients; $46 \%)$ compared with the younger population $(23 / 69 ; 33.3 \%)(p=0.262)$. This was also the case for all grades of diarrhea, which occurred in $42.9 \%$ of the older group and $26.1 \%$ of the younger group ( $p=0.221)$. The differences in the levels of more severe HFS (grades 2 and 3) between both groups [14/69 younger patients $(20.3 \%)$ vs. $22 / 63$ older patients (34.9\%); $p=0.059$ ]. Serious diarrhea (grades 2-4) was more prevalent during capecitabine therapy in older $(21 / 63 ; 31.7 \%)$ than younger patients $(12 / 69$; $17.4 \%)(p=0.044)$. Life-threatening diarrhea with dehydration (grade 4) was only seen in three patients in the older group. The two age groups did not differ significantly in their hematological adverse effects, such as neutropenia [15/69 younger patients (21.7\%) vs. 15/63 older patients (25.4\%); $p=0.522]$.

Dose adjustments of the capecitabine therapy because of adverse effects were initiated for 79 of the 132 patients included in the study (59.8\%), with adjustments being made more frequently to the dosage for older patients $(52 / 63 ; 83 \%)$ than the younger group $(27 / 69 ; 39 \%)(p=0.011)$. The main reasons for these dose adjustments were HFS, diarrhea, bone

\begin{tabular}{|c|c|c|c|}
\hline Measured variables & $\begin{array}{l}\text { Age } \leq 70 \text { years } \\
N=69\end{array}$ & $\begin{array}{l}\text { Age }>70 \text { years } \\
N=63\end{array}$ & $\begin{array}{l}\text { Difference } p \\
\text { value }\end{array}$ \\
\hline Age (years), mean & 57.0 & 74.1 & \\
\hline Female, $n(\%)$ & $31(44.9)$ & $24(38)$ & 0.426 \\
\hline Dosage (mg), mean (SD) & $1772(455)$ & $1869(387)$ & 0.476 \\
\hline Karnofsky Perfomance Scale (KPS) 60 & $0(0)$ & $1(1.8)$ & \\
\hline KPS 70 & $4(8.9)$ & $8(14.3)$ & \\
\hline KPS 80 & $12(26.7)$ & $16(28.6)$ & \\
\hline KPS 90 & $22(48.9)$ & $27(48.2)$ & \\
\hline KPS 100 & $7(15.6)$ & $4(7.1)$ & \\
\hline KPS $(0-100)$, median & 87.1 & 84.3 & 0.546 \\
\hline Renal function Cockcroft (mL/min), mean (SD) & $113.9(35)$ & $77(20.8)$ & 0.0001 \\
\hline Polypharmacy $^{\mathrm{a}}, n(\%)$ & $13(19.1)$ & $26(45.6)$ & 0.002 \\
\hline Charlson Comorbidity Index, mean & 5.27 & 6.0 & 0.007 \\
\hline Curative treatment intention, $n(\%)$ & $14(20.6)$ & $11(17.5)$ & 0.671 \\
\hline \multicolumn{4}{|l|}{ Type of tumor, $n(\%)$} \\
\hline Colon carcinoma & $45(65.2)$ & $44(77.8)$ & \\
\hline Breast carcinoma & $9(13)$ & $4(6.3)$ & \\
\hline Gastric carcinoma & $5(7.2)$ & $4(6.3)$ & \\
\hline Pancreas carcinoma & $3(4.3)$ & $2(3.2)$ & \\
\hline Other or unknown & $6(8.7)$ & $3(4.8)$ & \\
\hline \multicolumn{4}{|l|}{ Capecitabine treatment with: } \\
\hline Monotherapy capecitabine ${ }^{\mathrm{b}}, n(\%)$ & $23(33.3)$ & $20(31.7)$ & 0.03 \\
\hline Oxaliplatin/irinotecan $^{\mathrm{c}}, n(\%)$ & $15(21.7)$ & $14(22.2)$ & \\
\hline Bevacizumab, $n(\%)$ & $0(0)$ & $12(19)$ & \\
\hline 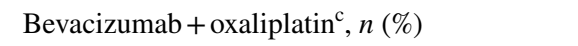 & $19(27.5)$ & $12(19)$ & \\
\hline Other or unknown, $n(\%)$ & $11(16.2)$ & $5(7.9)$ & \\
\hline
\end{tabular}

$S D$ standard deviation

${ }^{a}$ Definition of polypharmacy is five or more drugs

${ }^{\mathrm{b}}$ Dosage $1250 \mathrm{mg} / \mathrm{m}^{2}$

${ }^{\mathrm{c}}$ Dosage $800 \mathrm{mg} / \mathrm{m}^{2}$ 
Table 2 Adverse effects in patients

\begin{tabular}{lccc}
\hline Measured adverse effects, $n(\%)$ & $\begin{array}{l}\text { Age } \leq 70 \text { years } \\
(N=69)\end{array}$ & $\begin{array}{c}\text { Age }>70 \text { years } \\
(N=63)\end{array}$ & $p$ value Chi square \\
\hline Hand-foot syndrome & & & 0.262 (all grades) \\
Grade 0 & $46(66.7)$ & $34(54)$ & \\
Grade 1 & $9(13)$ & $7(11.1)$ & \\
Grade 2 & $8(11.6)$ & $15(23.8)$ & \\
Grade 3 & $6(9.7)$ & $7(11.1)$ & 0.059 (severe grades) \\
Mild (grade 0 and 1) & $55(79.7)$ & $41(65.1)$ & 0.221 (all grades) \\
Severe (grade 2 and 3) & $14(20.3)$ & $22(34.9)$ & \\
Diarrhea & & & \\
Grade 0 & $51(73.9)$ & $35(55.6)$ & \\
Grade 1 & $6(8.7)$ & $7(11.1)$ & \\
Grade 2 & $4(5.8)$ & $6(9.5)$ & \\
Grade 3 & $8(11.6)$ & $12(19)$ & \\
Grade 4 & $0(0)$ & $3(4.8)$ & 0.636 (severe grades) \\
Mild (grade 0 and 1) & $57(82.6)$ & $42(66.2)$ & \\
Severe (grade 2-4) & $12(17.4)$ & $21(31.7)$ & \\
Hematology & & & \\
Grade 0 & $54(78.3)$ & $48(76.2)$ & \\
Grade 1 & $10(14.5)$ & $9(14.3)$ & \\
Grade 2 & $5(7.2)$ & $4(6.3)$ & \\
Grade 3 & $0(0)$ & $2(3.2)$ & \\
Mild (grade 0 and 1) & $64(92.8)$ & $(7.2)$ & \\
Severe (grade 2 and 3) & & & \\
\hline & & & \\
\end{tabular}

According to National Cancer Institute Common Terminology Criteria Toxicity Scale Version 2

Hand-foot syndrome: $0=$ none; $1=$ skin changes or dermatitis without pain (e.g., erythema, peeling); $2=$ skin changes with pain not interfering with function; $3=$ skin changes with pain interfering with function

Diarrhea: $0=$ none; $1=$ increase of $<4$ stools/day over pretreatment; $2=$ increase of $4-6$ stools/day or nocturnal stools; $3=$ increase of $\geq 7$ stools/day or incontinence or need for parenteral support of dehydration; $4=$ physiological consequence requiring intensive care or hemodynamic collapse

Bone marrow: $0=$ normal for age; $1=$ mildly hypocellular or $<25 \%$ reduction; $2=$ moderately hypocellular or $25-50 \%$ reduction; $3=$ severely hypocellular or $50-75 \%$ reduction; $4=$ aplasia

\begin{tabular}{lcll}
\hline Adverse effect & $\begin{array}{l}\text { Dosage adjustment for patients } \\
\text { aged } \leq 70 \text { years, } n / N(\%)\end{array}$ & $\begin{array}{l}\text { Dosage adjustment for patients } \\
\text { aged }>70 \text { years, } n / N(\%)\end{array}$ & $p$ value \\
\hline Hand-foot syndrome & $9 / 69(13)$ & $18 / 63(29)$ & 0.073 \\
Diarrhea & $5 / 69(7)$ & $14 / 63(22)$ & 0.034 \\
All adverse effects leading & $27 / 69(39)$ & $52 / 63(83)$ & 0.011 \\
\hline
\end{tabular}

Table 3 Reason for dose adjustment compared the incidence of dose adjustments in these groups and investigated the reasons for these changes. We found that the incidence of HFS was not statistically different between the older and younger patients. Only the incidence of severe diarrhea differed between patients of different ages, being more common in the older group, and more dose adjustments were made for the older patients. These observations confirm the observed increased
This study compared the adverse effects of capecitabine therapies in real-world patients aged over 70 years with their incidence in younger patients. Secondarily, we marrow depression, and renal impairment. These results are shown in Table 3.

\section{Discussion}


incidence in the adverse effects of capecitabine in older patients reported in several other studies [13].

There are regional differences for the tolerability profiles of capecitabine, as was shown in a large retrospective safety analysis across the world. Similarly, the number of adverse effects related to fluoropyrimidine treatment is considerably higher in USA than in the rest of the world, particularly in Europe and East Asia, which could be owing to genetic polymorphisms, differences in folate intake, or cultural differences [16]. Some patients may be more willing to accept toxicity and continue treatment. Our analysis found different levels of adverse effects in elderly patients than were reported in earlier studies [4, 5, 13]; for example, severe HFS (grade 3) was documented in 11\% of the patient population aged older than 70 years, which is somewhat less than the $21 \%$ of patients aged older than 65 years experiencing severe HFS reported in a previous randomized controlled trial undertaken by Cassidy et al. [10]. The number of patient records evaluated in the study was too small to evaluate the less common adverse effects of capecitabine.

We did not find a statistically significant difference in the occurrence of HFS, only a difference in the incidence of severe diarrhea; however, we did find a difference in the total number of dosage adjustments, which could suggest that oncologists tend to take more precautions in the elderly. It is possible that oncologists prescribe oral capecitabine to frailer older patients based on the good safety results of this therapeutic for elderly patients in earlier studies. However, a Dutch study demonstrated differences between hospitals and physicians regarding the safety ratings of chemotherapy. The variability reported for these differences increases with the age of the patients $[17,18]$. The reason for these differences are not clear but some researchers have hypothesized that the more patients a specialist has treated, the more comfortable this specialist becomes with their treatment, accepting more adverse effects. In addition, the age of the medical specialist is known to be important, as is the involvement of scientific research and multidisciplinary healthcare teams [19].

The performance status of the patients included in our study was slightly lower than in the earlier studies; here, the Karnofsky Performance Scale score was 87.1 (median) in the younger cohort and 84.3 (median) in the older cohort of patients, while Cassidy et al. reported a score of 88.3 (median), in the older population [10]. The Eastern Cooperative Oncology Group performance score reported in the $\mathrm{X}$-ACT study also seemed higher than the patients in our study would have scored [5]. This could be explained by the fact that certain patients are excluded from clinical trials, such as patients with central nervous system disease or psychiatric disorders, whereas in our study population, some of these more vulnerable patients were included. Better clinical studies including older and more vulnerable people are warranted [20].
Our findings are consistent with those of Kalsi et al., who showed that low-grade toxicity in older people can lead to treatment modification [21]. However, it is also possible that the differences in toxicity between the older and younger patients are so small because of the number of dosage adjustments made for the older cohort.

Despite a similar incidence in both age groups for all grades of HFS, the incidence of severe HFS was higher in older patients (35\% compared with $20 \%$ ) but did not reach a statistical difference. In a vast majority of older patients (83\% compared with $39 \%$ in the younger age group), adverse events led to dose adjustments. One explanation might be oncologists are wary of maintaining doses in older patients presenting with mild HFS or a mild but cumulative other toxicity.

Our study has several limitations. First, this is a retrospective study with inherent missing data and limitations with regard to causality. The team medical oncologist in our hospital scored the adverse effects in real life as they do in normal daily practice using a standardized adverse event form similar to those frequently used in prospective trials. In our cohort, patients were not tested for dihydropyrimidinedehydrogenase deficiencies, as is standard practice now; however, there is no reason to believe that patients with partial dihydropyrimidine-dehydrogenase deficiencies were not equally divided between the two age groups. The incidence of impaired dihydropyrimidine-dehydrogenase metabolism is up to $5 \%$ in the general population [22].

It was difficult to determine the exact causality of the adverse effects. The older patients in our study had a significantly lower renal function when measuring the estimated glomerular filtration rate (Cockcroft-Gault) 113.9 vs. $77 \mathrm{~mL} / \mathrm{min}$. Because capecitabine is predominantly eliminated renally, it is contraindicated in patients with severe renal impairment (creatinine clearance below $30 \mathrm{~mL} / \mathrm{min}$ ). The incidence of grade 3 or 4 adverse events was higher in our study than in the overall population [23]. In patients with mild renal impairment no dosage reduction is required; however, it is plausible that renal dysfunction leads to higher capecitabine blood concentrations and greater toxicity, requiring dose adjustment.

Because of the small numbers of patients, a regression analysis was not feasible. We cannot rule out that the adverse events in older people are related to other factors, such as comorbidities.

The strength of this study cohort is that it contains an adequate number of unselected older people representative of the older patients seen in daily practice. All patients were treated by the same medical staff in the same hospital, which should have prevented bias from differences in protocols and medical facilities.

Some adverse effects such as HFS are associated with better survival; however, in more vulnerable patients, they 
can also lead to a greater impact on quality of life [24, 25]. In a meta-analysis of 14 clinical trials, patients taking capecitabine who developed HFS had a longer overall survival than patients who did not develop HFS: 1100 days (95\% confidence interval 1007-1200) vs. 691 days, with a hazard ratio of 0.61 (95\% confidence interval 0.56-0.66) [24]. These data must be considered when deciding whether to reduce the dose of capecitabine because of HFS, if the goal of the treatment is prolonged survival. Further research and subgroup analyses in older patients are required to fully evaluate this outcome.

Our data show a significant difference in the incidence of moderate-to-severe diarrhea in the elderly. Diarrhea is an underestimated problem in elderly patients, frequently leading to dehydration, functional decline, and hospitalization [26].

\section{Conclusion}

This study shows that the data for toxicity and dosage adjustments can vary between daily practice and clinical registration studies, and that dosage adjustments can be made based on both clinical and subjective criteria following toxicity experience. In the future, more prospective studies involving elderly patients are needed. With adequate preplanned subgroup analysis and more attention to, and awareness of, low-grade toxicity, better (personalized) decisions should be possible.

\section{Compliance with Ethical Standards}

Funding No sources of funding were used to assist in the conduct of this study or the preparation of this article.

Conflict of Interest Michiel W. H. van Beek, Monique Roukens, Wilco C. H. Jacobs, Johanna N. H. Timmer-Bonte, and Cornelis Kramers have no conflicts of interest directly relevant to the content of this study.

Ethics Approval The study protocol was reviewed by the Radboud University Medical Center Institutional Review Board, which concluded that the study did not fall within the scope of the Medical Scientific Research Act because it was a retrospective observational study, without intervention and with no burden for the participants.

Informed Consent No written informed consent was needed.

Open Access This article is distributed under the terms of the Creative Commons Attribution-NonCommercial 4.0 International License (http://creativecommons.org/licenses/by-nc/4.0/), which permits any noncommercial use, distribution, and reproduction in any medium, provided you give appropriate credit to the original author(s) and the source, provide a link to the Creative Commons license, and indicate if changes were made.

\section{References}

1. Folprecht G, Cunningham D, Ross P, Glimelius B, Di Costanzo F, Wils J, et al. Efficacy of 5-fluorouracil-based chemotherapy in elderly patients with metastatic colorectal cancer: a pooled analysis of clinical trials. Ann Oncol. 2004;15(9):1330-8.

2. Goldberg RM, Tabah-Fisch I, Bleiberg H, de Gramont A, Tournigand C, Andre T, et al. Pooled analysis of safety and efficacy of oxaliplatin plus fluorouracil/leucovorin administered bimonthly in elderly patients with colorectal cancer. J Clin Oncol. 2006;24(25):4085-91.

3. Moertel CG, Fleming TR, Macdonald JS, Haller DG, Laurie JA, Goodman PJ, et al. Levamisole and fluorouracil for adjuvant therapy of resected colon carcinoma. N Engl J Med. 1990;322(6):352-8.

4. Efficacy of adjuvant fluorouracil and folinic acid in colon cancer. International Multicentre Pooled Analysis of Colon Cancer Trials (IMPACT) investigators. Lancet. 1995;345(8955):939-44.

5. Twelves C, Scheithauer W, McKendrick J, Seitz JF, Van Hazel G, Wong A, et al. Capecitabine versus 5-fluorouracil/folinic acid as adjuvant therapy for stage III colon cancer: final results from the X-ACT trial with analysis by age and preliminary evidence of a pharmacodynamic marker of efficacy. Ann Oncol. 2012;23(5):1190-7.

6. Andre T, Boni C, Mounedji-Boudiaf L, Navarro M, Tabernero J, Hickish T, et al. Oxaliplatin, fluorouracil, and leucovorin as adjuvant treatment for colon cancer. $\mathrm{N}$ Engl $\mathrm{J}$ Med. 2004;350(23):2343-51.

7. Chionh F, Lau D, Yeung Y, Price T, Tebbutt N. Oral versus intravenous fluoropyrimidines for colorectal cancer. Cochrane Database Syst Rev. 2017;7:CD008398.

8. Pasetto LM, Monfardini S. The role of capecitabine in the treatment of colorectal cancer in the elderly. Anticancer Res. 2006;26(3B):2381-6.

9. Walko CM, Lindley C. Capecitabine: a review. Clin Ther. 2005;27(1):23-44.

10. Cassidy J, Twelves C, Van Cutsem E, Hoff P, Bajetta E, Boyer M, et al. First-line oral capecitabine therapy in metastatic colorectal cancer: a favorable safety profile compared with intravenous 5-fluorouracil/leucovorin. Ann Oncol. 2002;13(4):566-75.

11. Lou Y, Wang Q, Zheng J, Hu H, Liu L, Hong D, et al. Possible pathways of capecitabine-induced hand-foot syndrome. Chem Res Toxicol. 2016;29(10):1591-601.

12. van Erning FN, Razenberg LG, Lemmens VE, Creemers GJ, Pruijt $\mathrm{JF}$, Maas HA, et al. Intensity of adjuvant chemotherapy regimens and grade III-V toxicities among elderly stage III colon cancer patients. Eur J Cancer. 2016;61:1-10.

13. Feliú J, Escudero P, Losa F, Bolaños M, Vicent JM, Yubero A, et al. A phase II study of capecitabine (X) in elderly patients (p) as 1st line treatment for patients (pts) with advanced or metastatic colorectal cancer (MCRC). J Clin Oncol. 2004;22(14 Suppl.):3567.

14. Bosse D, Vickers M, Lemay F, Beaudoin A. Palliative chemotherapy for patients 70 years of age and older with metastatic colorectal cancer: a single-centre experience. Curr Oncol. 2015;22(5):e349-56.

15. Khattak MA, Townsend AR, Beeke C, Karapetis CS, Luke C, Padbury R, et al. Impact of age on choice of chemotherapy and outcome in advanced colorectal cancer. Eur $\mathrm{J}$ Cancer. 2012;48(9):1293-8.

16. Haller DG, Cassidy J, Clarke SJ, Cunningham D, Van Cutsem E, Hoff PM, et al. Potential regional differences for the tolerability profiles of fluoropyrimidines. J Clin Oncol. 2008;26(13):2118-23.

17. Keikes L, van Oijen MGH, Lemmens V, Koopman M, Punt CJA. Evaluation of guideline adherence in colorectal cancer treatment 
in the Netherlands: a survey among medical oncologists by the Dutch Colorectal Cancer Group. Clin Colerectal Cancer. 2018;17(1):58-64.

18. van Steenbergen LN, Rutten HJ, Creemers GJ, Pruijt JF, Coebergh JW, Lemmens VE. Large age and hospital-dependent variation in administration of adjuvant chemotherapy for stage III colon cancer in southern Netherlands. Ann Oncol. 2010;21(6):1273-8.

19. Hershman DL, Buono D, McBride RB, Tsai WY, Neugut AI. Influence of private practice setting and physician characteristics on the use of breast cancer adjuvant chemotherapy for elderly women. Cancer. 2009;115(17):3848-57.

20. Hurria A, Dale W, Mooney M, Rowland JH, Ballman KV, Cohen $\mathrm{HJ}$, et al. Designing therapeutic clinical trials for older and frail adults with cancer: U13 conference recommendations. J Clin Oncol. 2014;32(24):2587-94.

21. Kalsi T, Babic-Illman G, Fields P, Hughes S, Maisey N, Ross P, et al. The impact of low-grade toxicity in older people with cancer undergoing chemotherapy. Br J Cancer. 2014;111(12):2224-8.
22. Lunenburg CA, Henricks LM, Guchelaar HJ, Swen JJ, Deenen MJ, Schellens JH, et al. Prospective DPYD genotyping to reduce the risk of fluoropyrimidine-induced severe toxicity: ready for prime time. Eur J Cancer. 2016;54:40-8.

23. Ilich AI, Danilak M, Kim CA, Mulder KE, Spratlin JL, Ghosh $\mathrm{S}$, et al. Effects of gender on capecitabine toxicity in colorectal cancer. J Oncol Pharm Pract. 2016;22(3):454-60.

24. Sargent DJ, Goldberg RM, Jacobson SD, Macdonald JS, Labianca $\mathrm{R}$, Haller DG, et al. A pooled analysis of adjuvant chemotherapy for resected colon cancer in elderly patients. N Engl J Med. 2001;345(15):1091-7.

25. Ogawa C, Morita M, Omura A, Noda T, Kubo A, Matsunaka $\mathrm{T}$, et al. Hand-foot syndrome and post-progression treatment are the good predictors of better survival in advanced hepatocellular carcinoma treated with sorafenib: a multicenter study. Oncology. 2017;93(Suppl. 1):113-9.

26. Schiller LR. Diarrhea and malabsorption in the elderly. Gastroenterol Clin North Am. 2009;38(3):481-502. 